\title{
Médiévales
}

Langues, Textes, Histoire

74 | printemps 2018

Chanter la Croisade albigeoise

\section{Jeunesse et légitimité dynastique dans le chant de l'Anonyme La mortz o la terra}

\section{Laurent Macé}

\section{(2) OpenEdition}

1 Journals

\section{Édition électronique}

URL : https://journals.openedition.org/medievales/8523

DOI : $10.4000 /$ medievales.8523

ISSN : 1777-5892

Éditeur

Presses universitaires de Vincennes

\section{Édition imprimée}

Date de publication : 15 juillet 2018

Pagination : 83-98

ISBN : 978-2-84292-837-7

ISSN : 0751-2708

\section{Référence électronique}

Laurent Macé, « Jeunesse et légitimité dynastique dans le chant de l'Anonyme La mortz o la terra », Médiévales [En ligne], 74 | printemps 2018, mis en ligne le 15 juillet 2019, consulté le 23 avril 2022. URL : http://journals.openedition.org/medievales/8523; DOI : https://doi.org/10.4000/medievales. 8523 
Laurent Macé

\section{Jeunesse et légitimité dynastique dans le chant de l'Anonyme La mortz o la terra}

Le fils de la Vierge leur envoya, pour les réconforter, avec un rameau d'olivier, un bonheur, une lumineuse étoile qui se lève le matin sur la montagne : le vaillant jeune comte, la lumière et l'héritier. (Chanson de la Croisade albigeoise, laisse 201, v. 45-48.)

La composition de l'Anonyme, qui fait suite à la première partie de la Chanson de la Croisade albigeoise due à Guillaume de Tudèle ${ }^{1}$, est une chanson d'espoir, une vive espérance qui doit être incarnée par les plus dignes représentants de la jeunesse indigène. Ses vers tendent à construire une poétique du regard qui sacralise le chevalier naissant; son projet est au cœur d'une esthétique centrée sur l'homme neuf. Les princes joves ne manquent pas dans sa production, certains y ont même la part la plus belle, si ce n'est la première. à l'image de Raymond VI de Toulouse, principal dirigeant compromis par les affaires du «negocium pacis et fidei $^{2}$ », la vieille génération des comtes méridionaux semble avoir perdu tout crédit en ce début de $\mathrm{XIII}^{\mathrm{e}}$ siècle ; elle est la cible de regards réprobateurs, qu'ils soient pontificaux ou royaux ${ }^{3}$. La carence des chefs est d'autant plus criante que le

1. Sur cette seconde partie de la Chanson de la Croisade albigeoise, cf. l'Avant-propos de ce dossier thématique.

2. Excommunié en 1207 par le pape Innocent III, Raymond VI l'est, de nouveau, le 10 juillet 1210 (concile de Saint-Gilles), puis le 6 février 1211 (concile de Montpellier). G. Gouiran a souligné combien la seconde partie de la Chanson présente ce comte comme un prince faible, ne faisant guère l'unanimité et bien incapable de sauver un Paratge menacé par les clercs et les Français (G. GoUIRAN, «L'Anonyme frappe "ses" trois coups : l'atmosphère à Muret avant la grande bataille », dans V. BELTRAN, T. MARTínEZ et I. CAPDEVILA éd., 800 anys després de Muret. Els trobadors i les relacions catalonooccitanes, Barcelone, 2014, p. 131-148).

3. À l'automne 1212, le plan de paix proposé à Rome par le roi Pierre II d'Aragon prévoyait l'abdication de Raymond VI en faveur de son fils; le projet fut rejeté par le pape 
roi d'Aragon est mort ${ }^{4}$. Il faut donc un sang nouveau, des forces vives sur lesquelles nul soupçon d'hérésie ne peut avoir prise. Des jeunes gens qui pourront garantir une longue vie à Paratge, cet honneur ou cette noblesse qui est au cœur de cette Canso, et aux autres valeurs que partagent les élites guerrières de la sphère occitanophone. Ils pourront y parvenir - l'auteur le croit et ne cesse de le clamer - car ils sont des héritiers légitimes, en raison de leur noble naissance et de la terre qu'ils tiennent depuis toujours des mains de leurs aïeux.

Dans l'œuvre de l'Anonyme, trois fils nés dans la dernière décennie $\mathrm{du} \mathrm{XII}^{\mathrm{e}}$ siècle, trois figures de jeunes princes - tous issus d'une bien prestigieuse ascendance - sont mis en relief. Tout d'abord, Raimondet, le très attendu Raymond VII (1222-1249) ; puis l'aîné du comte de Foix, le futur Roger Bernard II (1223-1241) ; enfin, le premier enfant mâle du comte de Comminges, Bernard V (1225-1241). Cette même génération, appelée à exercer prochainement et simultanément le pouvoir au sein de ces trois grandes principautés, semble déterminée à accomplir son héroïque destin. Mais il lui faut pour cela restaurer un ordre du monde compromis par l'intrusion, injustifiable dans le paysage politique méridional, non pas de la croisade mais «des clercs et des Français », des hommes motivés par une certaine cupidité territoriale.

Ces jeunes gens, notamment Raimondet, apparaissent donc à des moments-clés de l'œuvre, tout particulièrement dans trois grands épisodes notables : le concile du Latran, le siège de Beaucaire et le siège de Toulouse ${ }^{5}$. Ces séquences permettent d'illustrer leurs droits légitimes et les vertus qu'ils incarnent en raison même de la noble origine qui est la leur. Les deux compagnons du Toulousain, Roger Bernard et Bernard, sont traités distinctement ou ensemble en fonction de la représentation qu'en veut donner l'Anonyme dans sa vision idéale des relations féodo-vassaliques ayant alors cours dans le Midi ; mais aussi selon le rapport personnel qu'il cherche à entretenir avec l'un ou l'autre de ces êtres en devenir. Ce lien, plus ou moins privilégié avec les trois hommes, invite à s'interroger sur le

Innocent III au mois de mai 1213 (M. Alvira CABRER, L. MACÉ, D. SMITH, « Le temps de la Grande Couronne d'Aragon du roi Pierre le Catholique. À propos de deux documents relatifs à l'abbaye de Poblet (février et septembre 1213) », Annales du Midi, 265 (2009), p. 5-22 (p. 9).

4. L'Anonyme, très au courant des faits, commence son œuvre dans un contexte politique bien singulier. Le dimanche 27 janvier 1213, à Toulouse, les aînés des comtes de Toulouse, de Foix et de Comminges ont, avec leur père, prêté serment de fidélité au roi Pierre II d'Aragon (M. Alvira CABrer, L. MACÉ, D. SMith, « Le temps de la Grande Couronne... », p. 10-11). Devenus « orphelins » de leur souverain, en raison de sa disparition sur le champ de bataille de Muret, ces trois jeunes princes ne peuvent dorénavant compter que sur eux-mêmes.

5. Pour une analyse détaillée de ces moments, $c f$. M. RAGUIN, Lorsque la poésie fait le souverain. Étude sur la Chanson de la Croisade albigeoise, Paris, 2015, p. 162-165, 169-176, 433-442, 512-524. 
mécénat dont aurait joui, à différents moments de sa production, l'auteur de la seconde partie de la Chanson.

\section{La métamorphose du " comte jeune "}

Quand l'Anonyme reprend l'œuvre de Guilhem de Tudèle, il mentionne pour la première fois l'aîné du comte de Toulouse juste après le désastre de Muret (12 septembre 1213) ${ }^{6}$. Après un séjour à la cour de son oncle Jean sans Terre $^{7}$, il revient sur le continent à l'automne, avec l'objectif de rejoindre son père afin d'aller avec lui au concile du Latran dont la réunion est prévue pour novembre $1215^{8}$. Pour l'essentiel, les propos abordent la question de l'héritage dont les jeunes gens risquent d'être dépossédés ${ }^{9}$.

Devant l'assemblée œcuménique de l'Église, les défenseurs des princes méridionaux désignent Raymond comme le fils du comte, c'està-dire avant tout comme son héritier. Pour le comte de Foix, il s'agit de plaider la cause de son seigneur et de son fils («mos senher, e sos filhs »), lequel ne saurait être considéré comme un éventuel coupable pouvant « suffrir dezeretament ${ }^{10}$ ». Pour l'archidiacre de Lyon, l'adolescent a pour lui la noblesse de sa lignée : l'antique chaîne raimondine ne saurait être rompue par la privation de l'héritage ${ }^{11}$. Ce que confirme l'archevêque de Dublin ${ }^{12}$ pour qui Raimondet est « l'onratz nebs del rei » Jean, il est « filhs legismes », issu du «milhor linatge » : il ne peut donc perdre la terre de son

6. «Pueih issic de sa terra, e sos filhs ichamens » (laisse 141, v. 17). Pour les passages de la Chanson cités, on se reportera à La Chanson de la Croisade albigeoise, éd. E. MARTINCHAвот, t. I-III, Paris, (1931-1961) 1960-1973 (2 éd.).

7. L. MACÉ, Les Comtes de Toulouse et leur entourage, XII-XIII siècles. Rivalités, alliances et jeux de pouvoir, Toulouse, 2000, p. 68.

8. «[...] el e son pauc filh [...] son intratz en Roma» (laisse 142, v. 13-14). Sur le contexte autour de l'assemblée romaine, voir la récente analyse de M. ALVIRA, «La convocation du quatrième concile du Latran et la croisade contre les Albigeois », dans D. J. SMith et J. BIRD éd., Outremer. Studies in the Crusades and the Latin East, Turnhout, sous presse.

9. Raymond de Roquefeuil prend le parti du fils de Trencavel, le défunt vicomte de Béziers et de Carcassonne : on doit rendre terra et eretat à un enfant orphelin (laisse 146, v. 31-46). Il faut souligner que la jonction formelle entre le texte de Guilhem de Tudèle et celui de son Continuateur se fait logiquement autour du verbe dezeretar (M. RAGUIN, « Le personnage du roi d'Aragon dans la Chanson de la Croisade albigeoise », Cultura Neolatina, 72 (2012), p. 53-85, en particulier p. 71).

10. Laisse 144 , v. 16-26.

11. «Ja l'ondratz filhs del comte, tant es de rics parens, / No er dezetatz senes clam longamens » (laisse 148, v. 55-56).

12. P. DufFy défend la thèse que le prélat anglais qui intervient en faveur du jeune Raymond n'est pas Walter de Grey, archevêque d'York, mais Henri de Londres, archevêque de Dublin, un proche du roi Jean sans Terre : «Le comte d'Ulster et la croisade contre les Albigeois », Annales du Midi, 285 (2014), p. 5-27 (p. 19-20). 
père ni celle de sa mère et encore moins devenir un pécheur en péril, errant seul comme un mauvais larron ${ }^{13}$.

Innocent III le reconnaît bien, il est le neveu du roi («sieu nebot »), ce Raymond est le fils du comte ${ }^{14}$. Mais après le départ du père, à partir de la laisse 152, lorsque le jeune prince de dix-huit ans se trouve dans l'environnement du pape, il passe de l'état de fils à celui d'enfant (« efans ») ${ }^{15}$. Les dialogues qui s'établissent alors prennent la forme d'une relation parent/enfant, le pontife paraît incarner une sorte de père moral, comme s'il était le bienveillant protecteur d'un prince en devenir («Filhs, so ditz l'Apostolis », v. 24). Il se substitue symboliquement au géniteur biologique sur lequel les soupçons de compromission avec l'hérésie sont bien trop lourds. Il est donc de son devoir de lui donner divers conseils pour espérer être un bon gouvernant qui saura défendre son droit ${ }^{16}$. Mais «l'enfant» argue justement devant son « senher » qu'il n'a pas de terre ${ }^{17}$, un seigneur considéré ici à la fois comme supérieur ecclésiastique (père de tous les fidèles) et comme pourvoyeur de biens et garant du droit, ainsi que doit l'être un honnête et véritable seigneur féodal.

À la fin de cet entretien qui a singulièrement pris une dimension programmatique, voire prophétique ${ }^{18}$, l'enfant est devenu un quasi-comte aux yeux de l'Anonyme ${ }^{19}$. Retrouvant enfin son père, il est certes à nouveau « fils » quand tous deux arrivent en Avignon, et c'est également ainsi que le voit Isnard Audiguier, le représentant des hommes de la ville rhodanienne ${ }^{20}$. Mais le véritable changement d'état s'opère sur un chemin de Provence (entre Salon et Avignon), à travers une sorte d'adoubement verbal - entendu ici dans le sens d'une reconnaissance collective, d'une aptitude à exercer le pouvoir. Avec cette particularité que ce rituel de passage est effectué par un parrain qui n'est autre qu'un représentant du monde courtois et guerrier, le chevalier troubadour Gui de Cavaillon ${ }^{21}$. Dans une forme littéraire qui

13. Laisse 150, v. 6-19. Cf. aussi L. MACÉ, «Raymond VII of Toulouse : The Son of Queen Joanna, "Young Count" and Light of the World», dans M. BULL et C. LÉGLU éd., The World of Eleanor of Aquitaine. Literature and Society in Southern France between the Eleventh and Thirteenth Centuries, Woodbridge, 2005, p. 137-156.

14. Laisse 150 , v. 36 ; laisse 151 , v. 57 et v. 60.

15. Laisse 152 , v. 1, 14, 17, 38, 51. Il était déjà dénommé ainsi, juste auparavant, par son père (laisse 151, v. 33). Une telle désignation vise à présenter le futur comte comme un être qui a encore besoin d'être placé sous une tutelle protectrice : pour les hommes du XIII ${ }^{\mathrm{e}}$ siècle, le passage de la pueritia à l'adulescentia se situe en général entre 12 et 15 ans.

16. M. RAGUIN, Lorsque la poésie fait le souverain..., p. 170-171.

17. Laisse 152, v. 18, 38, 51, 58.

18. M. RAGUIN, Lorsque la poésie fait le souverain..., p. 162-165.

19. «E1 coms issit de Roma » (laisse 152, v. 66). Ibid., p. 174-175.

20. Laisse 153 , v. 10 et v. 19. Sur l'identification de Isnard/Arnaud Audiguier, cf. L. MACÉ, Les Comtes de Toulouse..., P. 111.

21. Sur Gui de Cavaillon, voir M.AURELL, « Le troubadour Gui de Cavaillon (vers 1175vers 1229) : un acteur nobiliaire de la croisade albigeoise », dans Les Voies de l'hérésie : 
s'apparente fort à une tenso, ce dernier s'adresse directement à celui que l'Anonyme baptise le «comte jeune », le prince du futur ${ }^{22}$. Ce passage est capital et l'enjeu est de taille pour l'auteur : il s'agit de discuter de ce qui relève de Paratge, véritable socle d'un système de valeurs et d'un mode de sensibilité partagés par les élites du monde occitanophone ${ }^{23}$. La réponse du « coms jove » est à la hauteur des attentes de l'aristocratie ; Paratge sera préservé, rien ne changera car « grand est mon droit, juste est ma cause ${ }^{24} »$.

Le père et l'adolescent ( «tos ») reviennent en Avignon aux cris de «Tholosa » : c'est la dernière fois qu'ils apparaissent ensemble dans le cadre précis de leur relation de parenté. Et c'est en « coms joves » que Raymond entre en Venaissin pour y accomplir son destin ${ }^{25}$. Ayant reçu le soutien d'un certain nombre de vassaux de la région, il achève de sortir de sa chrysalide. Il est devenu pour l'Anonyme le successeur déclaré de Raymond VI ; fier de sa lignée, conscient de son rang, de sa charge, de ses devoirs, il est à l'instar de ses ancêtres «comte, duc et marquis ${ }^{26}$ ». Il y a bien alors deux comtes, «lo coms e.l coms joves ${ }^{27} »$. Partant pour la péninsule Ibérique, le vieux prince passe symboliquement le relais à son héritier et confie Ramundetz à ses fidèles vassaux. Le "coms joves » a acquis plus qu'une légitimité de façade, il est fort dorénavant d'une reconnaissance juridique et d'une réelle autorité conjointe, puisqu'il est en mesure de sceller des mandements qu'il s'empresse d'expédier aussitôt à ses alliés afin de préparer sa première opération militaire ${ }^{28}$.

le groupe aristocratique en Languedoc (XI'-XIII ${ }^{e}$ siècles). Actes de la $8^{e}$ session d'Histoire Médiévale du Centre d'Études Cathares (Couiza, août 1995), Carcassonne, 2001, vol. 2, p. 9-36.

22. « Guis de Cavalho desobr' un caval ros / A dig al comte jove» (laisse 154, v. 6-7).

23. E. M. GHIL, L’Âge de Parage. Essai sur le poétique et le politique en Occitanie au XIII siècle, New York/Berne/Francfort/Paris, 1989, p. 151-204.

24. «E es tant grans mos dreitz e la mia razos » (laisse 154, v. 25).

25. Laisse 154, v. 34 et v. 59.

26. «Coms dux e marques, del linatge n'Anfos; le coms dux qu'es tozetz » (laisse 154, v. 82 ; laisse 155, v. 1). Sur cette triple titulature, $c f$. L. MACÉ, Les Comtes de Toulouse..., p. 288-292.

27. Laisse 155 , v. 4.

28. Laisse 155 , v. 9, v. 32 ; «E1 coms joves tramet cartas e sageletz (v. 47). Sur cette capacité juridique à sceller, $c f$. L. MACÉ, La Majesté et la croix. Les sceaux de la maison des comtes de Toulouse (XII'-XIII siècle), Toulouse, 2018. 


\section{Le héros de Beaucaire}

Beaucaire constitue l'acte de naissance du futur comte Raymond VII ${ }^{29}$. Étroitement lié à son histoire personnelle ${ }^{30}$, le site castral participe à une sorte d'avènement anticipé de ce que devrait être son principat. Quand le jeune homme arrive, alors que le siège de la forteresse se prépare, il est aussitôt désigné, au début de la laisse 156, comme le comte naturel (« lo coms naturaus »). Natura renvoie au lieu d'origine, à l'appartenance territoriale, c'est-à-dire aux racines et à la naissance (natus), toutes deux ordonnées par le dessein divin (l'ordre de la nature) ; c'est aussi un terme qui exprime la pérennité d'un illustre lignage dont on descend ${ }^{31}$. Le prince vient donc pour rejoindre «ses amis de cœur» («sos amics coraus »), les gens de Beaucaire dont il est en retour «notre seigneur de cœur » (« nostre senhor coraus ») ${ }^{32}$. Plus avant dans le siège, l'Anonyme donne la parole aux chevaliers croisés pour insister sur ce lien infrangible qui unit l'enfant du pays à la ville rhodanienne ${ }^{33}$.

La dimension agonistique de Raymond est une obligation sociale, elle participe de son accomplissement chevaleresque, elle manifeste sa prestigieuse origine ${ }^{34}$. Durant les différentes phases de cette opération militaire, le jeune homme montre sa maîtrise du sens tactique, prend les décisions qui s'imposent en se comportant en véritable chef expérimenté ${ }^{35}$. Il fédère autour de sa personne les dépendants de ses domaines et d'ailleurs

29. L. MACÉ, «Des eaux du Rhône au vin de Genestet. Beaucaire dans le dispositif politique des Raimondins », dans M. Bourin et M. ReBoul éd., Le Siège de Beaucaire, 1216. Pouvoir, société et culture dans le Midi rhodanien, à paraître.

30. Raimondet est né au bord du Rhône et c'est là qu'il fut juridiquement émancipé en février 1211 (L. MACÉ, Les Comtes de Toulouse..., p. 60 et 69). Cf. l'analyse de cet important épisode par M. RAGUIN, Lorsque la poésie fait le souverain..., p. 512-524.

31. Mais la natura ne suffit pas pour le jeune Raymond, il lui faut en plus être un bon seigneur et un bon chevalier pour exprimer des qualités qui viennent légitimer sa « nature » d'origine. Sur la notion de seigneur naturel, $c f$. G. MARTIN, « Le concept de "naturalité" (naturaleza) dans les Sept parties d'Alphonse X le Sage »,e-Spania, 5 (2008), [en ligne] $<$ DOI : 10.4000/e-spania.10753>.

32. Laisse 156, v. 5 et v. 9. E. Martin-Chabot traduit coraus par « dévoués », puis par « bien-aimé » (La Chanson..., t. II, p. 109). Je préfère insister ici sur la relation directe au cœur qui me paraît plus proche de l'esprit de partage exprimé par l'auteur. Et cela permet aussi d'évoquer de façon implicite l'idée de courage qui anime le seigneur légitime des lieux.

33. «Il n'est pas facile d'enlever un château à son seigneur légitime ("senhor natural"). Les beaucairois aiment le jeune comte ("comte jove") de tout leur cœur ("per fina amor coral") et ardemment, ils le préféreraient de beaucoup à Jésus-Christ » (laisse 169, v. 8-10).

34. Sur les attentes communes de l'élite combattante, $c f$. X. STORELLI, «Du mérite militaire et de la prouesse chevaleresque dans le monde anglo-normand au XII ${ }^{\mathrm{e}}$ siècle », dans F. Bougard, R. LE JAn et T. LIENHARD éd., Agôn. La compétition, Ve-XII siècle, Turnhout, 2012, p. 131-159.

35. «E lo coms fai barreiras de lhissas e de paus / E mes a Santa Pasca las mainadas comtaus ; / E dedins en la roca es lo naveis aitaus » (laisse 156, v. 37-39). 
(« Als baros de sa terra e als seus domengers / Per trastotas las terras ${ }^{36} »$ ). Dans les affrontements menés aux portes de Beaucaire, il n'hésite pas à s'exposer au sein de la mêlée ${ }^{37}$. La bravoure du «valens coms joves » sert ainsi d'exemple à son entourage de courageux guerriers qui voient en lui l'authentique restaurateur de Paratge $^{38}$. Cette capacité physique à défendre son droit, les armes à la main, montre aux yeux de tous qu'il est apte à recouvrer sa terre, celle qu'il tient de ses ancêtres ${ }^{39}$. L'enjeu des combats dépasse le simple cadre militaire, la valeur du prince joue comme une ordalie, elle justifie une fois de plus une continuité dynastique qui a toujours prévalu : "Bientôt va être décidé qui est celui qui doit avoir la terre et la gouverner désormais », déclare l'un de ses fidèles avant de lancer l'assaut ${ }^{40}$.

À deux reprises, l'Anonyme prête des paroles acerbes à certains membres du conseil de Simon de Montfort. dans un premier temps, les propos achoppent sur la question de la légitimité induite par le sang et le lignage. Alain de Roucy parle de l'orgueil, de l'arrogance et de la tromperie (« engans ») qui règnent parmi les siens. En face d'eux, le contraste est grand quand se trouvent énoncées de nobles vertus irriguées par la qualité de l'antique origine du prince : «si le comte est jeune, un enfant ["es jove ni tozetz ni efans"], il est bien doué et bon et grand ["de bona natura e bos e bels e grans"], il a des ressources et de bons défenseurs [...]. Il est bien d'une race ["ben de lhinatge"] à se grandir et à se pousser en avant, car Richard fut son oncle et Bertrand son parent ["sos parens"]. On peut le traiter de pécheur, moi je dis que c'est un enfant ["enfans"] qui, au premier coup, a amené le six ${ }^{41}$. L Les dés en sont jetés ! Plus loin dans le siège, lors d'une nouvelle consultation, c'est avec irritation que Hugues de Lacy lance : « celui qui conquiert la terre ["terra"] et s'empare de la demeure d'autrui au détriment de la justice ["dreitura"] et en employant la tromperie ["engan"] et la méchanceté [“mal"], celui-là perd le fief ["onor"] qu'il a conquis, revenus et capital ${ }^{42} »$. Tout le vocabulaire des relations féodo-vassaliques est ainsi convoqué pour dénoncer le caractère illégal de l'expédition armée conduite par Simon de Montfort.

Tout logiquement, au moment de la reddition des assiégés et du recul patent de Montfort, Raimondet devient «lo coms de Tholoza» en titre ${ }^{43}$. Il

36. Laisse 159 , v. 21-22.

37. Laisse 161 , v. 30 ; « si la situation devient critique pour vous, je serai là, en homme qui sait bien venir à l'aide, pour prendre ma part du péril » (laisse 163, v. 35-36).

38. Laisse 167 , v. 37-42.

39. «Dieus vos [...] vol cobretz la terra que tengon lh'ancessor [...]. De totas partz escridan : "Tolza !" li milhor, / Per l'ondrat filh del comte qui recobra sa honor, / E intran a Belcaire » (laisse 163, v. 6-8 et 77-79).

40. Laisse 161 , v. 38-39.

41. Laisse 160 , v. 34-35 et 38-41.

42. Laisse 169 , v. 16-18.

43. Laisse 170, v. 46. 
hérite donc de la triple titulature [«coms, dux e marques »] que portaient avant lui son père et son grand-père ${ }^{44}$. Son lignage parle pour lui : «parce qu'il est vaillant ["valens"] et sage ["savis"], habile ["adreitz"] et courtois ["cortes"], de la plus excellente lignée ["milhor linatge"] et apparenté [“del ric parentes"] à la puissante maison de France et à celle du bon roi d'Angleterre ["del barnatge de Fransa e del bo rei Engles"] ${ }^{45}$. » Pourtant, ces qualités ne parviennent pas à masquer un goût étrange dans la bouche de certains proches du nouveau parangon de la noblesse méridionale. Certes, Montfort a plié mais il a pu repartir avec ses hommes et l'ensemble de ses forces, ce dont pâtissent les habitants de Toulouse lors de la brutale répression que connaît la ville en septembre 1216. Beaucaire, victoire ou demi-échec ? La question a sans doute taraudé l'esprit de certains ${ }^{46}$.

Par la suite, la figure princière du «senher dreituriers », qui vient défendre «la terra » et recouvrer «l'eres», prend une coloration à la fois christique et courtoise chez celui qui est devenu «comte» pour l'Anonyme ${ }^{47}$. L'arrivée triomphale à Toulouse, après la Pentecôte (3 juin 1218), de « l'astre de lumière » («clara estela ») est d'ailleurs un prélude à la mort de l'usurpateur, dont l'enseigne au lion s'abîme simultanément dans les eaux de la Garonne ${ }^{48}$. La ville même devient l'émanation de Pretz. e Paratge, du mérite et de l'honneur, et son jeune maître «fait reverdir le monde, le colore et redore ce qui avait été assombri ${ }^{49}$ ». Derrière les thèmes classiques de la si troubadouresque reverdie, le charisme naturel qui jaillit de la personne comtale évoque le vert de la jeunesse et l'or de la largesse ${ }^{50}$. Le combat de Baziège et la perspective d'un nouvel assaut sur Toulouse donnent encore l'opportunité à l'auteur de brosser le portrait d'un jeune dynaste prêt à défendre coûte que coûte son héritage et sa

44. Laisse 171, v. 6.

45. Laisse 171, v. 7-9. Cf. aussi avant la bataille de Baziège : « lo coms joves marques / Del linhatge de Fransa e del bo rei Engles » (laisse 210, v. 61-62).

46. L. MACÉ, " "La croix fait des progrès et le lion perd du terrain". Le siège de Beaucaire (fin mai-24 août 1216) », dans L. AlBARET et N. GOUZY éd., Les Grandes Batailles méridionales (1209-1271), Toulouse, 2005, p. 93-104 (p. 100-102).

47. Laisse 198, v. 12 ; laisse 210, v. 12. Cf. E. M. GHIL, L’Âge de Parage..., p. 179-181 ; M. RAGUIN, Lorsque la poésie fait le souverain..., p. 433-442.

48. « le fils de la Vierge leur envoya [...] une lumineuse étoile, l'étoile qui se lève le matin sur la montagne : le vaillant jeune comte, la lumière et l'héritier ["eretier"] [...]; le comte comme une fleur de rosier [...] tout joyeux, descendit au monastère de monseigneur saint Sernin » (laisse 201, v. 45-48, 61 et 64-65). Idem, plus loin, dans la bouche du consul maître Bernard qui l'appelle «notre lumière » (laisse 204, v. 117).

49. « lo valens coms joves, que 1 mon fa reverdir / E colora e daura que s sol escurzir » (laisse 208, v. 102-103).

50. Sur le vert assimilé à la jeunesse, voir M. PASTOUREAU, Vert. Histoire d'une couleur, Paris, 2013, p. 71-76. 
principauté $^{51}$. Sur le mode du débat - qui n'est pas sans rappeler un thème abordé par certaines chansons de geste -, l'échange argumenté qu'il nourrit plus loin avec l'un de ses mentors, Pelfort de Rabastens ${ }^{52}$, est un plaidoyer convaincant pour un droit féodal qui parle en faveur d'un vassal bien mal honoré par son suzerain ${ }^{53}$. L'agression de Louis, fils de Philippe Auguste, délie le Toulousain, descendant des comtes et des rois, de l'allégeance due à son souverain. L'affaire est donc sans issue, il faut à nouveau se battre contre «l'orgueil de France [«l'orgolh de Fransa »] ${ }^{54} »$. Raimondet et son frère Bertrand tiendront donc la barbacane Villeneuve contre Louis et ses faux « porteurs de bourdon ${ }^{55} »$.

\section{Le vaillant Roger Bernard de Foix}

Après l'épisode fondateur de Beaucaire, un autre jeune surgit dans le paysage de la chanson. Il s'agit du fils du comte de Foix ${ }^{56}$. Comme le Toulousain, il doit défendre son espace hérité quand celui-ci se trouve menacé par les sombres prétentions des croisés. En février 1217, contre l'avis des instances romaines, Simon de Montfort s'en prend au comte de Foix et assiège le castrum de Montgrenier que défend son fils. Toutefois, l'«adreit Roger Bernat» est contraint de traiter avec son adversaire ${ }^{57}$. Mais ce n'est que partie remise. Six mois plus tard, le comte Raymond VI prépare depuis le Couserans son retour à Toulouse ${ }^{58}$. Il demande conseil à ses « amics privatz ». Juste après Bernard IV de Comminges, intervient le jeune prince : il encourage son seigneur à retrouver son « heretatge » et à

51. « il nous est possible de leur faire payer cher nos patrimoines ["las nostras heretatz"] » (laisse 211, v. 4).

52. Sur Pelfort de Rabastens, $c f$. L. MACÉ, Les Comtes de Toulouse..., p. 108-109.

53. Laisse 213 , v. 37-80.

54. Laisse 214 , v. 1.

55. Laisse 214, v. 60-64 ; laisse 213, v. 64.

56. Un autre fils, mais illégitime, appelé Loup de Foix, apparaît à trois reprises dans l'œuvre de l'Anonyme. Il est blessé au cours d'un affrontement durant le siège de Toulouse ; il figure aux côtés de son frère quand celui-ci se dirige vers le Lauragais (hiver 1219); il prend la parole juste avant la bataille de Baziège (laisse 195, v. 122 ; laisse 210, v. 43 ; laisse 211, v. 55-57).

57. Laisse 180, v. 17-26. L'anonyme reste volontairement discret sur cet épisode qui $a$ contrario est davantage décrit par Pierre des Vaux-de-Cernay, l'hagiographe de Simon de Montfort (Hystoria albigensis, chap. LXXXIV, § 589-590).

58. Il peut compter sur le soutien de Roger II, membre d'une branche collatérale des comtes de Comminges, qui détient la vicomté de Couserans et le comté de Pallars Sobirà. Il permet à Raymond VI de rassembler en Pallars des hommes recrutés en Catalogne et en Aragon ; certains sont d'ailleurs des vétérans qui souhaitent venger leur suzerain tué à Muret (M. Alvira CABrer, 12 de Septiembre de 1213. El Jueves de Muret, Barcelone, 2002, p. 537). 
relever Pretz e Paratges en luttant activement contre ses ennemis ${ }^{59}$. Paroles suivies d'effets puisque l'héritier des Foix prend la tête de l'avant-garde qui se dirige droit vers Toulouse, ses armoiries bien mises en évidence sur les gonfanons déployés ${ }^{60}$. L'occasion de montrer ses qualités militaires lui est donnée lors d'un engagement contre Joris, un chef de bande dont il abat victorieusement l'un des compagnons d'armes ${ }^{61}$. Et quand la ville est reprise, ce sont à nouveau les enseignes qui expriment visuellement les revendications armées et armoriées de cette princière jeunesse issue de la « terra » ancestrale. Elles flottent au-dessus de tous ces héritiers mais aussi des dépossédés - les chevaliers « faidits »-qui encadrent et soutiennent le vieux comte de Toulouse. C'est une telle image, savamment mise en scène, que perçoit avec inquiétude Alix, l'épouse de Simon de Montfort, depuis le château Narbonnais, l'antique résidence des Raymond ${ }^{62}$.

Pour l'essentiel, l'action déployée par Roger Bernard est souvent scandée sur un ton épique. Lorsque le prince combat près de la porte Montoulieu, sa présence et le déploiement de ses couleurs rassurent tout le monde dans la mêlée. Son cri de guerre est associé à celui des comtes raimondins ${ }^{63}$. Le palé d'or et de gueules (un écu orné de bandes verticales jaunes et rouges) rappelle à tous les armoiries du défunt Pierre II d'Aragon, dont les Foix sont des parents éloignés ${ }^{64}$. Toujours prompt à intervenir les armes à la main, Roger Bernard ne cesse de prendre part aux conseils comtaux et à donner son avis pour organiser au mieux la défense de la ville de Toulouse et de ses ponts sur la Garonne ${ }^{65}$. Des paroles aux gestes,

59. Laisse 181, v. $29-34$.

60. Laisse 181, v. 84-86.

61. Laisse 182 , v. 8-12.

62. "C'est le comte Raymond, qui revendique Toulouse ["qui Toloza requier"], et Bernard de Comminges, que j'aperçois aux premiers rangs, je reconnais sa bannière et son gonfalonnier ["la senheira el seu gomfanonier"]; il y a aussi Roger-Bernard, fils de Raymond-Roger [...] et les chevaliers "faidits" et les légitimes héritiers ["li dreit eretier"] » (laisse 183, v. 30-35).

63. « Roger-Bernard est venu au milieu de la mêlée, avec toute sa compagnie, qu'il mène et conduit ; sa présence raffermit les courages, aussitôt qu'il y fut reconnu. Messire Pierre de Durban, le seigneur de Montégut, lui portait son enseigne, dont la vue les a ranimés. Ayant mis pied à terre, il s'est placé au premier rang ; on cria et proclama : "Foix et Toulouse !" ["Foig e Tholoza"] » (laisse 184, v. 30-36).

64. L. MACÉ, La Majesté et la croix..., p. 385-386. Sur la place du comte de Foix et celle du représentant du roi, Dalmas de Creixell, dans le dispositif littéraire et idéologique de l'Anonyme, voir M. RAGUIN, « La Catalogne et les Catalans vus de la Chanson de la Croisade albigeoise : au-delà de la question du seul roi d'Aragon », dans V. BELTRAN, T. MARTínEZ et I. CAPDEVILA éd., 800 anys després de Muret..., p. 221-246 (p. 236, p. 243-246).

65. «Voici Roger-Bernard qui parle gracieusement et doucement ; parce qu'il est aimable et sage, qu'il est valeureux et sensé ["gentils e savis e a valor a sen"], qu'il est fils du bon comte qui possède et défend Foix, il discourt à merveille et leur dit en souriant » (laisse 197, v. 5-8 et suivants). «le bon et aimable Roger-Bernard avertit sur-le-champ le Chapitre et les communaliers » (laisse 198, v. 63-64). Au conseil, avec les «baros secretz », convoqué 
il n'y a qu'un pas qu'il franchit sans ambages ${ }^{66}$. Sa participation active à la défense de la capitale raimondine est sans cesse soulignée ${ }^{67}$. Elle fait de lui l'un des hommes les plus en vue aux yeux des élites toulousaines ${ }^{68}$. Et c'est naturellement dans l'un des épisodes les plus évocateurs d'espoir, la victoire remportée à Baziège, que le prince fuxéen - entouré de ses proches - s'illustre avec honneur aux côtés de Raimondet dont il est le principal soutien ${ }^{69}$.

Un tel rôle dans les situations guerrières et vassaliques, une présentation si positive de l'implication militaire dans la défense des intérêts raimondins, une si grande place dans le dispositif idéologique de l'auteur, s'expliquent en partie par la relation de mécénat qui a été établie avec le jeune prince. L'Anonyme le déclare lui-même : c'est le «preux Roger Bernard qui me rehausse et me donne de l'argent ${ }^{70} »$. Mais il ne semble pas être le seul concerné. Plus que jamais, la situation l'exige, la convergence d'intérêts des futurs comtes passe par l'axe Foix-Toulouse-Comminges ${ }^{71}$.

par Raymond VI pour déterminer l'action à mener contre la chatte, le comte de Comminges s'exprime, puis Roger Bernard enchaîne avec vigueur (laisse 203, v. 74-79).

66. «Ce fut Roger-Bernard qui construisit les premières assises [...] ; Roger-Bernard donne ses ordres, guide et organise la résistance » (laisse 197, v. 33 ; v. 82).

67. Début juin 1218 , il défend la barricade devant la place Montaigon (laisse 200, v. 2226). Puis dans la scène finale, lors de la distribution des sites stratégiques à protéger, Roger Bernard a pour mission de défendre la barbacane de Lascrosses en compagnie de quelques « faidits » de la vicomté de Carcassonne (laisse 214, v. 28-33).

68. "Quand mon seigneur le comte et messire Roger-Bernard et le comte de Comminges et messire Dalmas étaient dans cette ville avec nos autres concitoyens, le puissant et outrecuidant comte de Montfort restait enfermé dans ses tentes », rappelle le magistrat municipal maître Bernard (laisse 204, v. 106-110).

69. Il intervient en Lauragais avec son père et son frère Loup de Foix (laisse 210, v. 42 43). Puis Roger Bernard, « doué de toutes les qualités », exprime sa joie à se battre et rappelle la fidélité due à Raimondet avant de conduire la première ligne rassemblant, contre la troupe de Foucaud de Berzy, une partie des « faidits » du Carcassès (laisse 210, v. 86-89; laisse 211, v. 24-31 et v. 48-50).

70. «El pros Rotgiers Bernatz, que m daura e esclarzis », « de qui je reçois or et éclat » traduit l'éditeur (laisse 194, v. 64). Sur cette possibilité « de rétribution financière et de promotion sociale », $c f$. M. RAGUIN qui relève une idée équivalente à la fin de la chanson (laisse 214, v. 28-29) : « le bon Roger-Bernard, qui restaure ceux qui ont subi des pertes ("que restaurals perdens") » (Lorsque la poésie fait le souverain..., p. 77-78). Il faut noter qu'il en est dit de même de Raimondet, «qui redonne éclat et splendeur à ceux qui ont été ou sont spoliés [Que restaura Paratge [...] E colora e daura los perdutz ells perdens] » (laisse 214, v. 61-62). Est-ce là aussi une allusion au probable mécénat prodigué par le jeune comte, même si la gratitude du poète ne paraît point dans ce vers ? (contra M. RAGUIN, Ibid., p. 172).

71. Quoi de plus efficace que de mettre dans la bouche d'un prince croisé, comme le comte de Soissons, des propos sur la légitimité des droits appartenant aux seigneurs indigènes per linatge ? (laisse 202, v. 72-89). 


\section{Bernard le parent loyal}

C'est également au moment du retour de Raymond VI qu'apparait pour la première fois Bernard, le fils du comte de Comminges. Participant au conseil restreint du prince, il prend la parole juste après Roger Bernard de Foix pour signifier à son tour sa fidélité et sa loyauté à la dynastie raimondine : il se battra aux côtés des comtes pour qu'ils puissent recouvrer Toulouse et tous leurs biens ancestraux ${ }^{72}$. De fait, comme il l'avance peu après avec enthousiasme, la fraîche victoire contre la troupe de Joris est annonciatrice de la prochaine reprise de la ville ${ }^{73}$. C'est là qu'il s'illustre, notamment en combattant à proximité du château Narbonnais, secteur sensible car s'y trouve la résidence toulousaine des comtes de Comminges ${ }^{74}$. Sa belle action lui permet d'obtenir les louanges de l'Anonyme ${ }^{75}$. Et quand la résistance s'organise pour défendre la cité en prévision du retour de Simon de Montfort (octobre 1217), père et fils arborent leurs armoiries à côté de celles de leur parent raimondin ${ }^{76}$. Aussi, dans le tumulte des combats, les cris de guerre de ce conglomérat princier sont-ils une fois de plus associés ${ }^{77}$. Ce qui a pour effet immédiat d'impressionner certains compagnons du chef militaire de la croisade ${ }^{78}$.

72. Laisse 181 , v. $35-41$.

73. Laisse 182, v. 21-24.

74. C. Higounet, Le Comté de Comminges de ses origines à son annexion à la couronne, Toulouse, 1949, p. 104. La Chanson mentionne également cet édifice : «l'ostal del comte de Cumenge » (laisse 173, v. 9).

75. «Messire Bernard de Comminges s'y est bien comporté ; c'est lui qui, avec sa belle compagnie, vaillant et avisé, a occupé et tenu, du côté du Château, où était leur convoi, les débouchés et les passages ; aussi les louanges et le mérite doivent-ils lui en être accordés » (laisse 184, v. 57-61).

76. «E mantas de maneiras demonstran lor senhals : / Las doas crotz vermelhas e l'ensenha comtals. » L'éditeur de la Canso y voit celles du comte de Comminges et de son parent Roger, vicomte de Couserans (La Chanson..., t. III, p. 13, n. 8). Mais il est plus probable que ce soient les bannières de deux représentants de la dynastie comtale : Bernard porte son étendard quand il entre à Toulouse avec Raymond VI, et son père le rejoint peu de temps après (laisse 185, v. 63-64). Les armoiries des Bernardins portent une croix pattée qui s'inspire directement de l'insigne et des couleurs de la milice du Temple. Ces comtes sont les soutiens actifs d'un ordre militaire religieux qui lutte, à leurs yeux, pour la seule et vraie croisade qui doit exister (L. MACÉ, « Sceau du miles conversus. Entre l'idéal cistercien et le modèle templier (seconde moitié du XII ${ }^{\mathrm{e}}$ siècle) », dans D. CARRAZ et E. DeHOuX éd., Images et ornements autour des ordres militaires au Moyen Âge. Culture visuelle et culte des saints (France, Espagne du Nord, Italie), Toulouse, 2016, p. 133-140.

77. "On crie : "Toulouse ! voici que l'orgueil est humilié" et "Comminges !" pour le comte ; "Foix !" pour messire Roger-Bernard» (laisse 188, v. 43-44).

78. Ils sont cités comme les premiers alliés de Raymond VI : « le comte de Comminges, chevalier d'élite, le bon comte de Foix, son fils Roger-Bernard, messire Bernard de Comminges et tant d'autres seigneurs » (laisse 190, v. 123-125). Idem dans la bouche de Simon de Montfort (laisse 192, v. 33-35). 
Pour le distinguer de son père, Bernard IV, invariablement désigné comme « le comte de Comminges », l'Anonyme l'appelle toujours « Bernatz de Cumenge ». Dans la bouche de Raymond VI, il se trouve inséré dans le cercle de la parenté : il le désigne comme son «beau neveu » ( Bels neps ») dans la plupart de ses prises de parole ${ }^{79}$. Avec les comtes de Foix, il fait partie du petit cercle des « amics privatz » que consulte Raymond VI dans le cadre du conseil vassalique. Le jeune prince y est même présenté comme le porte-étendard des valeurs aristocratiques ${ }^{80}$. Il peut donc prendre la parole - après quelques avis formulés par des hommes d'expérience et par son pair, Roger Bernard de Foix - pour approuver le choix des positions militaires à tenir face aux entreprises croisées ${ }^{81}$; propos suivis d'effets puisque le jeune prince se retrouve au premier rang, menant les hommes de Toulouse au combat ${ }^{82}$.

Ayant fait ses preuves à Toulouse, après avoir loyalement servi son seigneur, le prince aguerri peut maintenant s'occuper de la défense de son patrimoine. Il quitte la ville assiégée pour aller lutter contre Joris qui s'ingénie à dévaster la campagne commingeoise ${ }^{83}$. Une laisse entière de 143 vers est consacrée à cet épisode qui met en valeur Bernard, «beau et bon et aimé de tous ${ }^{84} \gg$. Avec ses meilleurs barons, depuis le château de Saliès-du-Salat, il se lance dans une haletante poursuite du prédateur Joris qui vient de s'emparer de Saint-Gaudens et qui reconduit ses exactions en divers lieux. C'est dans ce contexte que l'héritier du comté s'adresse lui

79. Laisse 181 , v. 42 ; laisse 182 , v. 25. Voir également la relation de parenté existant entre Raymond VI et Bernard IV de Comminges (L. MACÉ, Les Comtes de Toulouse..., p. 93 96).

80. «E lo coms de Toloza a sos baros mandatz / E vol son cosselh pendre ab sos amics privatz / Cum defenda la vila [...] / E lo coms de Cumenge, ab complida lauzor / E lo rics coms de Fois, qui al pretz e la flor, / [...] E i esn Rogers Bernatz, ab sen e ab valor, / En Bernatz de Cumenge, qui porta l'auriflor / De pretz e de Largueza e de Gaug e d'Onor. » (laisse 190, v. 157-159 ; laisse 191, v. 3-8). Sur les « amics privatz », $c f$. L. MACÉ, « Amour et fidélité : le comte de Toulouse et ses hommes (XII ${ }^{\mathrm{e}}-\mathrm{XIII}{ }^{\mathrm{e}}$ siècles) », dans H. DÉBAX éd., Les Sociétés méridionales à l'âge féodal. Hommage à Pierre Bonnassie, Toulouse, 1999, p. 299304.

81. Laisse 191, v. 35-91.

82. «Messire Bernard de Comminges prend la tête et le commandement des hommes de la ville, pour qu'ils ne soient pas défaits, et puis il leur crie qu'on ne leur résisterait pas » (laisse 193, v. 28-30). Il est bientôt secondé par Roger Bernard qui l'aide à défendre un fossé menacé par l'offensive des croisés (v. 43-44). Puis, « hardi et allant de l'avant », il prend part à la sortie des Toulousains qui tentent de détruire la chatte de leurs adversaires (laisse 205, v. 52).

83. «Puis messire Bernard de Comminges a pris congé d'eux : il s'en va en Gascogne combattre ses ennemis, reprendre activement la guerre et courir sus à Joris » (laisse 194, v. 69-71) ; «Quant à messire Bernard de Comminges, il a besoin d'agir avec vigueur, car Joris chevauche à travers son domaine, envoie le défier et dévaste sa terre » (laisse 208, v. 107-109).

84. Laisse 209 , v. 5. 
aussi à ses hommes et leur promet d'assurer à la fois victoire, butin, gloire et restauration de Paratge, " car mieux vaut mourir avec honneur que vivre ainsi dans la honte ${ }^{85} »$. C'est une fois de plus les couleurs héraldiques de la troupe qui signalent aux usurpateurs, arrivés près de la barbacane de Meilhan, que le bon droit s'impose naturellement dans le ciel méridional ${ }^{86}$. Le violent combat tourne à la faveur du jeune prince ${ }^{87}$. Preuve a été faite qu'il a su se montrer à la hauteur de son rang. Le caractère assez laudatif de ce segment de l'œuvre, ainsi que d'autres passages tout aussi élogieux, laisseraient penser que le fils du comte de Comminges appartient au petit cercle des mécènes de l'Anonyme. Il est fort probable - même s'il n'intervient pas à la même hauteur de financement qu'un Roger Bernard de Foix - que ce prince, à l'instar de Raimondet, ait rétribué la relation de certains épisodes le mettant sur le devant de la scène ${ }^{88}$. Toujours est-il que, naturellement, le seigneur pyrénéen figure en bonne place dans le bouquet final de la chanson : en 1219, l'une des barbacanes protégeant les portes de Toulouse lui est confiée ${ }^{89}$. Comme les deux autres princes, il représente le porte-enseigne parfait des idéaux chevaleresques défendus par les hommes aptes à gouverner les terres de leurs ancêtres. Ici encore, l'utilisation rhétorique du personnage du jeune fait amplement écho à la loi de la nature qui veut que l'ancien se perde pour que puisse surgir le nouveau.

La mort ou la terre ${ }^{90}$. Telle semble être l'injonction suprême qu'adresse à ces trois jeunes princes le Continuateur anonyme de la Chanson. Et ses derniers vers s'achèvent sur une ultime prière qui convoque Dieu, le Christ, la Vierge, Saint-Sernin, « Razo, Dreitura, dreitz, forsa » et le « coms joves » afin de sauver Toulouse de l'orgueil de France ${ }^{91}$. La victoire à venir sera la traduction concrète du bien-fondé d'une bonne cause l'emportant sur une autre qui est vécue comme illégitime.

La fama, le renom d'une dynastie, le prestige acquis et personnel, quasi charismatique, est au cœur de la sensibilité aristocratique qui trouve ici ses porte-étendards. Au sein du «negocium pacis et fidei » s'ouvre donc un conflit de génération entre les pères compromis et les enfants garants de l'ordre établi. Cette société d'héritiers veut retrouver un temps mythique qui

85. Laisse 209, v. 60-77.

86. «Quand les Français virent les brillantes armoiries, la croix, le peigne, le taureau, la brebis et les autres enseignes des hardis barons, avec leurs belles troupes, qui les ont poursuivis, il n'est pas étonnant s'ils en furent ébahis. » (laisse 209, v. 85-89).

87. Laisse 210, v. 1-7.

88. Cf. supra n. 70 sur Raimondet qui restaure lui aussi les perdants...

89. «Bernard de Comminges, beau, aimable et gracieux, puissant, preux et sage, ardent et conquérant, et aussi Bernard de Comminges, son cousin, et Arnaud-Raymond d'Aspet tiennent avec courage [...] la barbacane qui vient d'être construite. » (laisse 214, v. 65-70).

90. Laisse 152, v. 55.

91. Laisse 214, v. 116-136. 
lui serait rendu, espère l'aube d'une ère nouvelle ouverte sur l'assomption du héros glorieux, un homme qui valoriserait une lignée dont la légitimité spatiale est bafouée par une croisade dirigée contre d'authentiques chrétiens $^{92}$.

Mais le combat se déroule aussi dans un cadre féodo-vassalique où il est sans cesse question de droit et d'usurpation, deux termes appartenant à une thématique du déshéritement si présente dans les conventions et autres serments de fidélité de ce temps. «Car Engans e Dreitura se son faitz cabalers / De tota aquesta guerra », déclare l'Anonyme au moment de l'arrivée des renforts français à Beaucaire ${ }^{93}$. Seul Dieu pourra mettre un terme à ce duel judiciaire qui va pourtant se poursuivre entre les fils, Raimondet et Amaury, à la mort de Simon de Montfort ${ }^{94}$. Mais l'issue ne pourra être qu'heureuse pour les détenteurs de l'héritage ancestral qui résistent encore et toujours à la dépossession, unis qu'ils sont sous la bannière des valeurs toulousaines. C'est en ce sens que, dans sa poétique de la guerre menée en commun, l'auteur chante une jeunesse qui financera encore les troubadours et la création épique romane, une jeunesse fédérée qui, selon son cœur, demeurera synonyme d'espoir et d'avenir. La contre croisade de l'Anonyme est une voie de salut placée sous le signe de la nécessité : il faut escompter que les jeunes princes sauront contenir les prochains assauts de l'an 1219. De fait, la capitulation, qui est acceptée dix ans plus tard, n'est que le début de la chronique d'une mort annoncée : la fin langagière qui se profile à l'horizon ne fait qu'amorcer la disparition inexorable de Paratge et de Joven ${ }^{95}$.

Laurent Macé - Université Toulouse-Jean-Jaurès, UMR 5136 Framespa

92. Dans le proche comté de Provence, c'est bien un tout jeune prince, Raymond Bérenger $\mathrm{V}$, qui va parvenir à fédérer l'aristocratie locale autour de sa personne, donnant ainsi naissance à une véritable principauté (T. PÉCOUT, « La mort de Pierre II et l'émergence d'une principauté en Provence », dans J. Le PotTier, J. Poumarède, C. MARqueZ, R. SouriaC éd., Le Temps de la bataille de Muret (12 septembre 1213), Actes du 61 congrès de la Fédération historique de Midi-Pyrénées, Montréjeau, 2014, p. 255-268).

93. Laisse 159 , v. 77-78.

94. "Que le fils de la Vierge, qui fait triompher le droit [“dreitz"] [...], sauvegarde Razo e Dreitura [...] Parce que le duel recommence entre Montfort et Toulouse, que le fils veut, à cause de la mort de son père, affermir son autorité » (laisse 208, v. 17-22).

95. M. BANNIARD, «Les enjeux d'une défaite: la mutation d'un paradigme», dans J. Le PotTIER et al.éd., Le Temps de la bataille de Muret..., p. 403-412 (p. 409-410). 


\section{Jeunesse et légitimité dynastique dans le chant de l'Anonyme La mortz o la terra}

Le Continuateur anonyme de la Canso accorde dans son œuvre une belle place aux jeunes princes méridionaux. Parmi eux, le fils du comte de Toulouse, le futur Raymond VII, apparaît comme la figure principale. Mais gravitent autour de lui d'autres fils, celui du comte de Foix et celui du comte de Comminges. Ces jeunes gens incarnent un espoir fort: celui d'une relève politique, non entachée par les soupçons de sympathie à la cause hétérodoxe; celui aussi du maintien d'un Paratge qui serait menacé par les agissements des "Français " et du clergé. En cela, l'auteur ne fait que développer un thème cher aux troubadours de son temps.

Bernard de Comminges, Chanson de la Croisade albigeoise, jeunesse, héritage, Paratge, Raymond VII de Toulouse, Roger Bernard de Foix.

\section{Youth and Dynastic Legitimacy in the Anonymous Song La mortz o la terra.}

The anonymous author of the continuation to the Canso devotes considerable attention to the young southern princes. The son of the Count of Toulouse, the future Raymond VII is foremost amongst them, but he is surrounded by other aristocratic scions including the sons of the Count of Foix and the Count of Comminges. These young princes embody a powerful sense of hope: hope in a new era of politics untainted by suspicions of sympathy for the heretics, and hope in the survival of a tradition of paratge presented as being under threat from the "French" and the clergy. In this respect, the author is simply expanding upon a theme already popular with contemporary troubadours.

Bernard of Comminges, Heritage, Paratge, Raymond VII of Toulouse, Roger Bernard of Foix, Song of the Albigensian Crusade, Youth. 